\title{
An Efficient Approach for Real Time Tracking of Intruder and Abandoned Object in Video Surveillance System
}

\author{
G. Prabhakar \\ Assistant Professor \\ Department of ECE \\ Syed Ammal Engineering College \\ Ramanathapuram, TamilNadu, India.
}

\begin{abstract}
Abandoned Object Detection and Intruder detection is one of the important tasks in video surveillance system. This paper proposes an integrated approach for the tracking of abandoned and unknown objects using background subtraction and morphological filtering. The aim of the approach is to automatically recognize activities around restricted area to improve safety and security of the servicing area by multiplexing hundreds of video streams in real time. The tracking module takes as input per camera tracking and recognition results and fuses these into object estimation. A novel algorithm for object tracking in video pictures, based on image segmentation is proposed. With the image segmentation all objects in images can be detected whether they are moving or not by using image segmentation results of successive frames. Consequently, the proposed algorithm can be applied to multiple movements. The algorithm was tested on real time video surveillance system and it produces very low false alarms and missing detection. This approach definitely provides security and detects the moving object in real time video sequence and live video streaming.
\end{abstract}

Keywords - Background Subtraction, Blob analysis, Morphological Filtering.

\section{INTRODUCTION}

Networks of video cameras are being installed in various applications such as surveillance of ATM machines and banks, disaster response, traffic management, environmental monitoring, etc. Recently the data collected by such networks is manually analyzed. So such task is extremely tedious and reduces the potential of the installed networks. . Even though some standards like PETS and i-Lids are available for detecting the abandoned objects, still there are some issues in video surveillance system. Hence it is essential to develop tools for analyzing the data collected from these cameras and summarizing the results to the user. Object Tracking and activity recognition are two fundamental tasks in this system. In this system, we develop methods for tracking and activity recognition in a distributed network of cameras. For many applications, for a number of reasons it is desirable that the video analysis tasks be decentralized. For example, there may be constraints of bandwidth, secure transmission, and difficulty in analyzing a huge amount of data centrally.

The cameras would have to act autonomously in such situations and also act as agents to make decentralized decisions. At the same time, however, the decisions of the cameras need to be coordinated so that there is a consensus on

\author{
B. Ramasubramanian \\ Assistant Professor \\ Department of ECE \\ Syed Ammal Engineering College \\ Ramanathapuram, TamilNadu, India.
}

the state (e.g., position, activity) of the target even if each camera is an autonomous agent. Thus, the cameras, analyze the raw data locally, exchange only refined information that is relevant to the collaboration, and reach a shared, global analysis of the scene as an autonomous one. Although there are a number of methods in video analysis that deal with multiple cameras, and even camera networks, distributed processing in camera networks has received very little attention and we will review the current state of the art in camera networks and will see that very few methods are capable of distributed analysis of video. On the other hand, distributed processing has been extensively studied in the multi-agent systems and Co-operative control literature. Methods have been developed for reaching consensus on a state observed independently by multiple sensors. However, there is very little study on the applicability of these methods in camera networks. The key concern for individuals is privacy and security. This paper proposes a video surveillance to achieve a better balance between both concepts: it protects privacy of the surveilled individuals, while giving support to efficient automated surveillance.

Beynon et al [5] defined an abandoned package as any stationary package away from anyone considered responsible for it. Bird et al [6] defined an abandoned object to be a stationary object that has not been touching a person for some time. Fernando et al [7]defined an abandoned object as a static non-human object which splits from a human. In all the above method, it is very difficult to detect the relationship of an abandoned object and its owner.

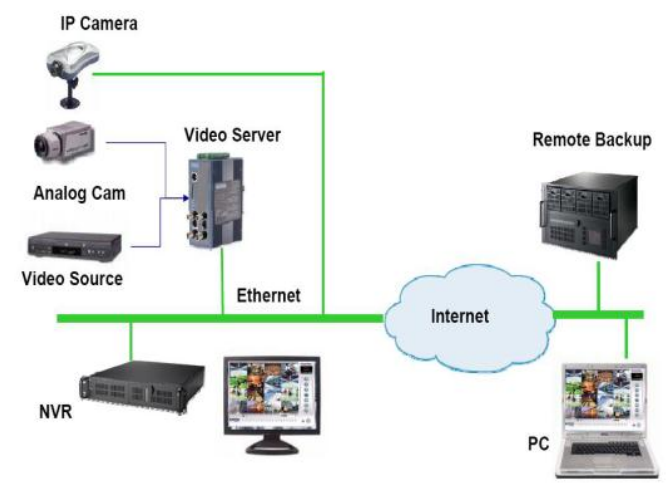

Fig 1: Block diagram of Autonomous Video Surveillance 
Figure 1 shows the multi camera tracking of objects. The tracked objects from each of the eight cameras are given as input to the system and output is estimated in the world coordinate system. Spatial registration is performed using percamera coplanar calibration and the camera streams are synchronized temporally across the network by a central video server.

The captured video signals are transmitted over a two-wire cable to the video server. It is situated in the control or equipment room close to the camera mounting. Additionally, an interruptible power supply unit should be used to compensate minor voltage fluctuations or short term power failures. The challenges of Autonomous Video Surveillance algorithm are Environmental Conditions such as Glitter of lamps/Daylight, Lightning, Shadows/ Reflections, Swaying trees / Moving Clouds, Rains / Snows and mechanical conditions such as Vibrations, Camera Quality, Noise under low lux and improper aperture under strong light.

\section{RELATED WORK}

Most of the proposed techniques for object detection rely on tracking information $[5,8,9]$ to detect drop-off events. As stated by Porikli [10], these methods are not well suited in complex scenes involving crowds and large amount of occlusions. To overcome these limitations, Porikli[10] proposed a single camera, non-tracking based system which makes use of two backgrounds for the detection of stationary objects. The two background are constructed by sampling the input video at different frame rates. But it is difficult to set the appropriate parameters to sample the input video for different applications.

The object video surveillance system [12] keeps track of background regions which are stored right before they are covered by an abandoned object. This approach fails when the static objects stays long enough in the scene, which makes the matching more difficult due to differences in Lighting.

Jianling et al [13] uses different background modeling technique with GMM for different scene type. In this approach, pixel variance of foreground in recent frames are found and pixels with robust variances lower than threshold is taken as suspected regions and connected component analysis is done and the abandoned object is detected using classifiers. To overcome this problem, Yingli et al [ ] proposed a method that uses more than one GMM to describe the statistics of each pixels. When an abandoned object is detected, GMM model A with high learning rate would show the static object faster than GMM model B with low learning rate. In method [7], texture information is integrated into the foreground mask computation to enhance Mixture of Gaussian method and region growing is used. Another approach is proposed which uses two backgrounds as in [previous] and uses radial reach filter to enhance the foreground mask.

The method which doesn't use background modeling is very less. A method is proposed in [] in which unattended and stolen object is detected based on the fusion of information derived from three fast detectors. However, it exploits different types of information like shape, similarity, Contour similarity, background similarity.
In this approach, we introduce a method to detect and track the objects in a video sequence and live video streaming. Background subtraction is the simplest method to detect the object. This section presents the thorough study on different tracking algorithms as well as on the use of cameras for capturing the video data set.

\section{COMPONENTS OF PROPOSED ALGORITHM}

This Algorithm consists of two parts

- Intrusion Detection

- Abandoned Object Detection

\subsection{Intrusion Detection}

Intrusion detection provides surveillance and automatically detects prohibited intrusion scenarios and can be used on stationary cameras. There are several modes for detection: Tripwire, Regional Entrance and Fence Trespassing.

In Regional Entrance, an alarm is produced when a person or vehicle moves within a restricted area. Detection can be defined to prohibit entrance from a specific direction while ignoring entry from other directions and can be defined independently for vehicle detection or stranger, each allowing distinct directional criteria.

In tripwire and fence trespassing, an alarm is produced, when a person or vehicle breaches a demarcation line of separation. Object Detection can be specified to prohibit any crossover or to allow movement in a single direction. Detection of Tripwire ignores movement in parallel to specified lines and only detects if the lines are crossed. The features of tripwire allows the definition of more than one line per scene and multiple segments per single line

\subsection{Abandoned Object Detection}

An alarm is produced when an item (package, debris, baggage, etc.) is deposited or appears in a controlled area. Additionally, detection can be configured to ignore items that are attended by a nearby person. It helps to reduce the need for patchy roving patrols and provides rapid detection and pre-alarm recording so that abandon luggage owners can be located more rapidly. It provides detection for disguised objects that may be overlooked by passing patrols or regarded as familiar.

When the baggage is carried into a scene by a person, baggage alone cannot be detected, but it can also be detected when the baggage is dropped or thrown into a scene from offcamera. Additionally, baggage can be detected even if it appears while the scene is temporarily blocked. The detection of abandoned object offers 3 levels to match the requirements of the scene, semi-crowded areas, unattended detection in sterile areas, as well as detection in crowded scenarios for the presence of stationary baggage. The ideal thing is for identifying the appearance of potential baggage bombs, railline obstruction hazards, traffic lane/runway debris, dumping/littering, fallen rocks etc.

\section{PROPOSED METHOD}

In this section we proposed method for real time object detection and tracking. The problem is divided into several steps. Here we will provide the algorithms for these steps. Also, we will discuss the use of multiple cameras to capture the video and create a video dataset. There will be a brief detail about the research issues with the use of multiple cameras. 


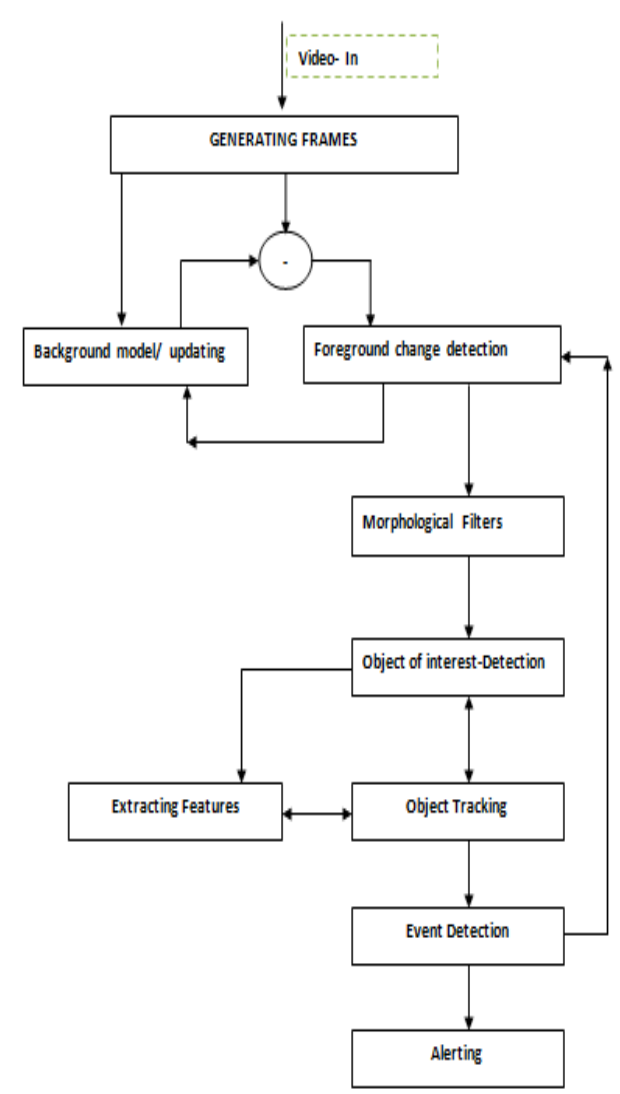

Fig 2: Video analytics system flow diagram

\section{A. Generating Frames:}

Here we have presented the algorithm to generate the image frames from video sequence. Steps are given as:

a) Read the video file using aviread ( ) for audio video interlaced file format or mmread ( ) for other supported file format.

b) Find the number of frames in video file using aviinfo ( ).

c) Convert the video into frames using frame2im ( ).

d) Write the converted frame on to the storage disk using imwrite ( ).

e) Repeat the step 3 and 4 up to the last frame of the video.

f) End

\section{B. Object Detection:}

Object detection is the process of finding out the area of interest as per user's requirement. Here we have proposed the algorithm for object detection using frame difference method (One of the background subtraction algorithms). Steps are given as:

a) Read all the image frames generated from the video, which are stored on a variable or storage medium.

b) Convert them into greyscale image using rgb2gray ( ) from coloured image.

c) Calculate the difference as $\mid$ frame $\mathrm{i}$ - frame $\mathrm{i}-1 \mid>\mathrm{Th}$.

d) If the difference is greater than a threshold $\left(r_{t h}\right)$, then the value is considered to be the part of foreground otherwise background.

e) Update the value of $i$ by incrementing with one.

f) Repeat the step 3 to 5 up to the last image frame.

g) End the process.

\section{Post Processing:}

The detected object in the previous phase may lead to have a problem of connectivity and it may also have some holes which may be useless for object representation. Therefore here we need to have some post processing which will reduce the problem of handling holes and the connectivity of pixels within object region. Mathematical morphological analysis is one of post processing approach which leads to enhance the segmented image in order to improve the required result. In the proposed method we have used the erosion and dilation iteratively so that an object will clearly appear in foreground while the rest useless blobs will be removed. Morphological operations are useful to obtain the useful components from the image. These components may be the object boundary, region, shape and skeleton etc.

Dilation: Dilation is an increasing transform, used to fill small holes and narrow chasm in the objects.

Erosion: Erosion as morphological transformation can be used to find the contours of the objects. It is used to shrink or reduce the pixel values.

D. Feature Selection:

The features like centroid of an object, height and width of an object are selected so that it is easy to plot the location of non rigid body/objects with frame to frame. The proposed method evaluates the centroid of detected object in each frame. It is assumed that after the morphological operations there will not be any false object. And then a centroid of the object in two dimensional frames can be calculated as the average of the pixels in $\mathrm{x}$ and $\mathrm{y}$ coordinates belonging to the object.

$$
\begin{gathered}
\mathrm{C}_{\mathrm{x}}=\sum_{i=1}^{N} \frac{X i}{N} \\
\mathrm{C}_{\mathrm{y}}=\sum_{i=1}^{N} \frac{X i}{N}
\end{gathered}
$$

\section{E. Object Representation:}

Here we are using centroid and the rectangular shape to cover the object boundary to represent the object. After calculating the centroid, find the Width $\mathrm{W}_{\mathrm{i}}$ and Height $\mathrm{H}_{\mathrm{i}}$ of the object by extracting the positions of pixels $\mathrm{Px}_{\max }$ and $\mathrm{Px}_{\min }$ which has the maximum and minimum values of $\mathrm{X}$ Coordinate related to the object. Similarly, for the Y coordinates, calculate $\mathrm{Py}_{\max }$ and $\mathrm{Py}_{\min }$

F. Trajectory Plot:

After the process of object detection using frame differencing method, the detected components are given as input to the tracking process to plot the trajectory. The frame differencing algorithm will give all the pixel values of the detected object. The centroid of the objects is calculated by using the equation 1 and 2. Here input will be the pixel values of the object and the output will be the rectangular area around the object. This process will calculate the centroid, height and width of the object for the purpose of trajectory plotting.

\section{G. Video Analytics Processing}

Segmentation is the process of detecting changes and extracting relevant changes for further analysis and qualification. Changed pixels from previous positions are referred to as "Foreground Pixels"; those that do not change are called "Background Pixels". The segmentation method used here is Background Subtraction. Image Pixels remaining after the background has been subtracted are the foreground pixels. The key factor which is used to identify foreground pixels by means of "Degree of change" in segmentation and can vary depending on the application.

The segmentation result is one or more foreground blobs. A blob is nothing but a collection of connected pixels. 
The process of classification is to qualify each blob and to assign a class label. This results in a broad categorization of each blob into sufficiently distinct classes such as vehicle, animal, person, etc., Classification may be done on a single frame or may use information over multiple frames. Small combination of properties of features of each blob is used to assign the class label. So these features need to be selected in a manner such that they provide sufficient discrimination between each valid class.

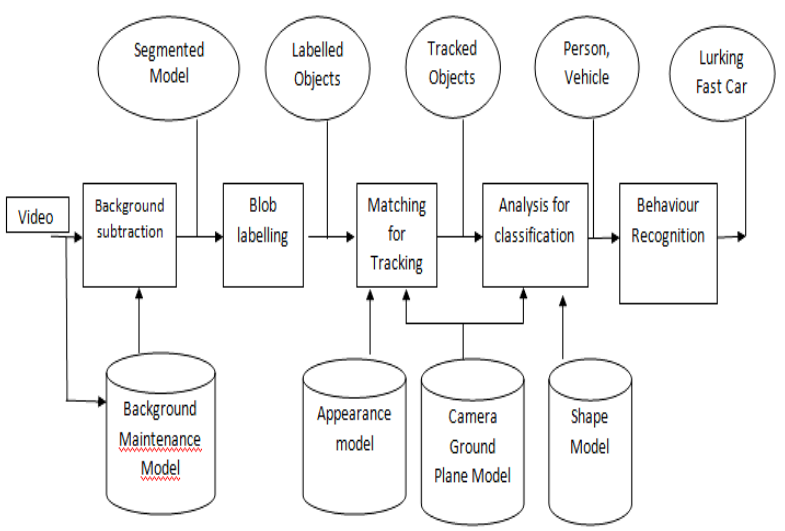

Fig 3: Video Analytics Processing Pipeline

Tracking of classified foreground blobs takes place over multiple frames as objects move through the field of view. Blob Tracking is a problem of blob association for each blob in a starting frame. The current position of that blob in successive frames needs to be identified. A trajectory can then be calculated for the object by connecting its position over multiple frames. The final step is the Activity Recognition which combines the results of classification \& tracking and correlating the tracks of multiple blobs to infer the activity occurring in the video. At instance, if two blobs corresponding to people progressively come closer, then it could be interpreted as converging people. There are two blobs, one corresponding to a vehicle and another corresponding to a person happens to merge, this could be interpreted as a person getting into a vehicle.

\section{SIMULATION AND RESULTS}

The simulation of Intruder detection and abandoned object detection is done by using MATLAB version 7.9 by considering background Image in a video of 200 frames. The simulation results for all the techniques are explained. Initially the moving objects in video images are tracked based on image segmentation, background subtraction and object detection techniques. The simulation results of the algorithms are shown below. Abandoned object detection: The input video sequence is shown as frames in Fig. 4. The abandoned object that are detected are shown in Fig 5a. The motion detection is identified and marked in yellow lines and the abandoned object found is marked in red color. The results obtained for Intrusion detection are shown in Fig.5b and the restricted area is marked in green color, when intruder passes in the restricted area the color of the bounding box changes to red.
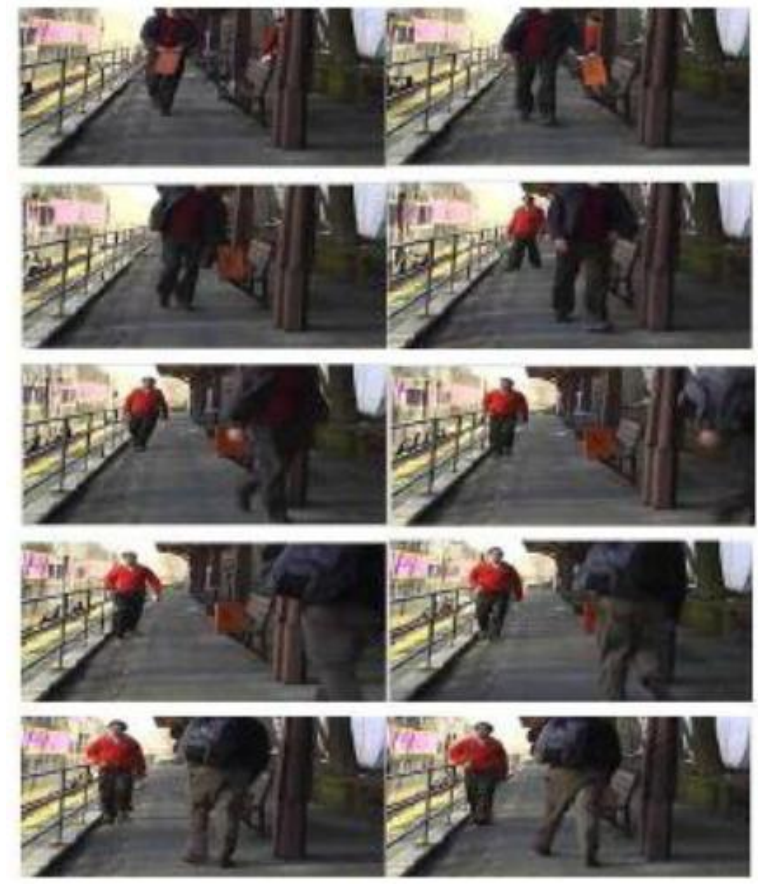

Fig 4: Input Frames of Video Sequence
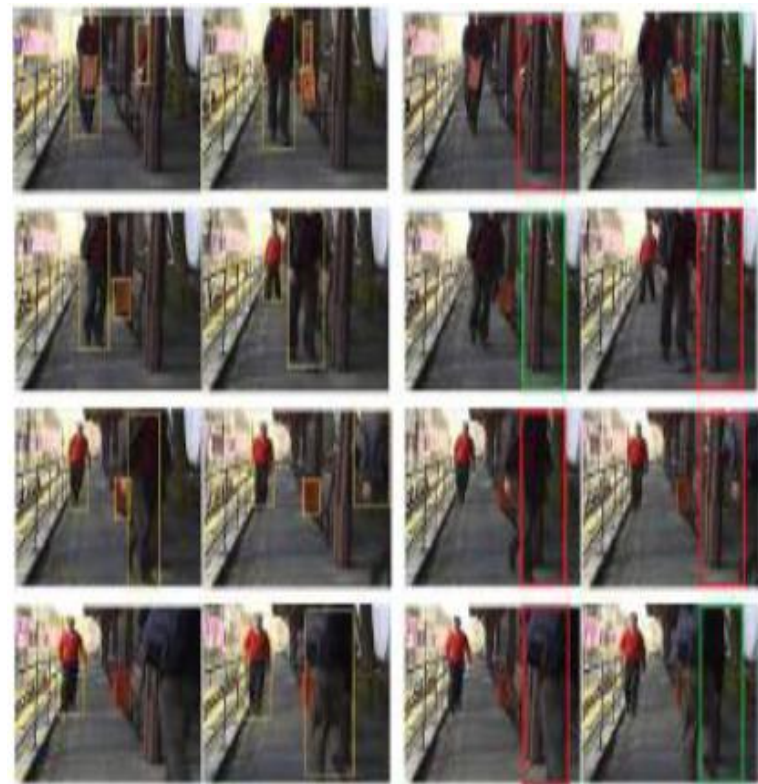

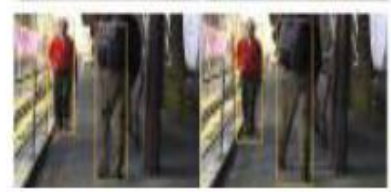

5(a)

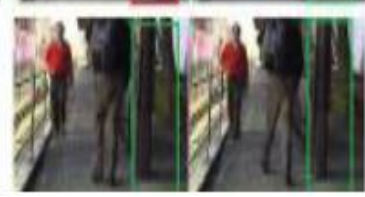

5 (b)
Fig5(a): Abandoned Object Detection

Fig5(b): Green indicates no Intrusion \& Intruder is denoted by RED box 


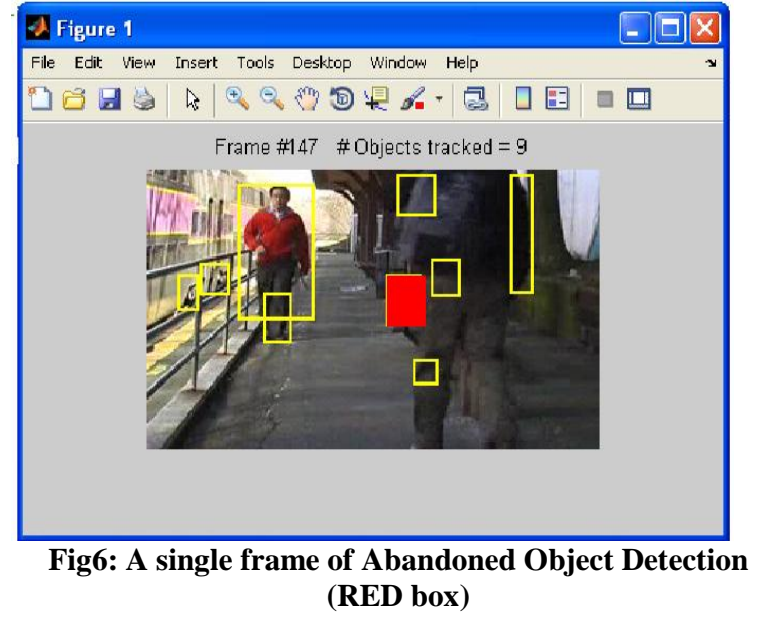

Figure 6 shows the result of abandoned object detection for a single frame. The RED box indicates the abandoned Object.

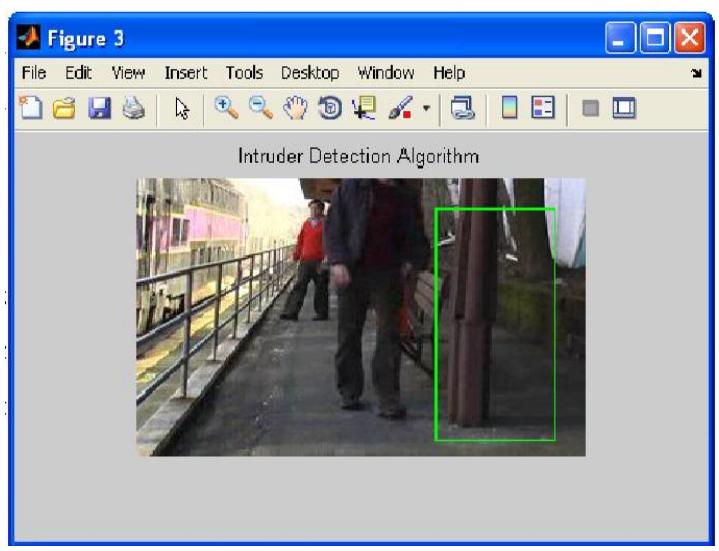

Figure 7(a). Green box indicates no Intrusion

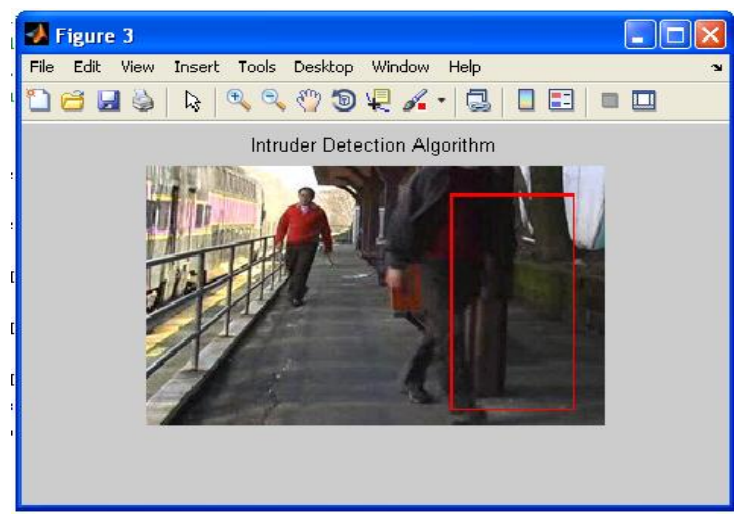

Figure 7(b). Intruder is indicated by RED box

Figure 7 (a) and (b) shows the result of intruder detection for a single frame. When there is no intruder, the bounding box is in Green color. When the intruder arrives, the color of the box changes to red indicating that there is a intruder in our surveillance area. We also evaluated our algorithm on i-LIDS dataset[19]

\section{CONCLUSION}

An object tracking algorithm for video pictures, based on image segmentation and pattern matching of the segmented objects between frames in a simple feature space is proposed.
The algorithm works very well for more complicated video pictures including rotating objects and occlusion of objects. If mistracking occurred at some frame by reason of occlusion, newly appearing or disappearing objects, the proposed algorithm could recover correct tracking after a couple of frames. The relative simplicity of this tracking algorithm promises that a DSP implementation is possible and already sufficient for real time applications with a few moving objects.

\section{REFERENCES}

[1] Schutte.J "Guide-way intrusion detection", in IEEE Vehicular Technology Magazine September 2009

[2] H.H. Liao, J.Y. Chang, and L.G. Chen, "A localized approach to abandoned luggage detection with foreground-mask sampling," in IEEE International Conference on Advanced Video and Signal Based Surveillance, 2008, pp. 132-139.

[3] X. Li, C. Zhang, and D. Zhang, "Abandoned objects detection using double illumination invariant foreground masks," in IEEE Int'l Conference on Pattern Recognition, 2010.

[4] Y. Tian et al., "Robust detection of abandoned and removed objects in complex surveillance videos," IEEE Trans. on Systems, Man, and Cybernetics, Part C: Applications and Reviews, vol.PP(99), pp. 1-12, 2010.

[5] M. Beynon, D. Hook, M. Seibert, A. Peacock, and D. Dudgeon, "Detecting Abandoned Packages in a Multicamera Video Surveillance System", IEEE International Conference on Advanced Video and Signal-Based Surveillance, 2003

[6] N. Bird, S. Atev, N. Caramelli, R. Martin, O. Masoud, N. Papanikolopoulos, "Real Time, Online Detection of Abandoned Objects in Public Areas", IEEE International Conference on Robotics and Automation, May, 2006.

[7] Ferrando.S, Gera.G, Regazzoni.C, "Classification of Unattended and Stolen Object in Video-Surveillance System", in IEEE International Conference on Video and Signal Based Surveillance, 2006. AVSS '06

[8] E. Auvinet, E. Grossmann, C. Rougier, M. Dahmane, and J. Meunier, "Left-luggage detection using homographies and simple heuristics," in PETS, 2006, pp. 51-58.

[9] S. Guler, J. A. Silverstein, and I. H. Pushee, "Stationary Objects in Multiple Object Tracking", IEEE International Conference on Advanced Video and Signal-Based Surveillance, London, UK , September 2007

[10] F. Porikli, Y. Ivanov, and T. Haga, "Robust Abandoned Object Detection using dual foreground" EURASIP Journal on Advances in Signal Processing, Volume 2008, Hindawi Publishing Corporation.

[11] D. Ramanan, "Using Segmentation to Verify Object Hypotheses", Proceedings of CVPR, 2007.

[12] P. L. Venetianer, Z. Zhang, W. Yin, A. J. Liptop, "Stationary Target Detection Using the ObjectVideo Surveillance System", IEEE International Conference on Advanced Video and Signal based Surveillance, London , UK, September 2007. 
[13] Jianting, Wen,Haifeng Gong, Xia Zhang and Wenze Hu , "Generative Model For Abandoned Object Detection",IEEE International Conference on Image Processing, 2009

[14] Ying-li Tian, Rogerio Feris, Arun Hampapur, "Robust Detection of Abandoned and Removed Objects in Complex Surveillance Videos", IEEE Transactions on Systems, Man, and Cybernetics,2010

[15] Bhargava, M.; Chia-Chih Chen; Ryoo, M.S.; Aggarwal, J.K ,"Detection of Abandoned Objects in Crowded Environments" Advanced Video and Signal Based Surveillance, 2007. AVSS 2007. IEEE Conference on Digital Object Identifier.

[16] Claudio Sacchi and Carlo S. Regazzoni, "Distributed Surveillance System for Detection of Abandoned Objects in Unmanned Railway Environments", IEEE Transactions on vehicular technology, 2000.
[17] Nathaniel Bird, Stefan Atev, Nicolas Caramelli, Robert Martin, Osama Masoud, Nikolaos Papanikolopoulos, "Real Time, Online Detection of Abandoned Objects in Public Areas", Proceedings of the 2006 IEEE International Conference on Robotics and Automation,Orlando, Florida - May 2006

[18] M. Beynon, D. Hook, M. Seibert, A. Peacock, and D. Dudgeon, "Detecting Abandoned Packages in a Multicamera Video Surveillance System", IEEE International Conference on Advanced Video and Signal-Based Surveillance, 2003

[19] i-LIDS Dataset for AVSS 2007, http://motinas.elec.qmul.ac.uk/pub/iLids. 